\title{
Current practice in Transvenous Lead Extraction in Latin-American: Latin-American Heart Rhythm Association Survey
}

\author{
Juan Carlos Diaz ${ }^{1}$, Jorge Romero ${ }^{2}$, Roberto Costa ${ }^{3}$, Alejandro Cuesta ${ }^{4}$, Luigi DiBiase ${ }^{5}$, \\ Isabella Alviz ${ }^{2}$, Alejandro Velasco ${ }^{5}$, Mohamed Gabr ${ }^{5}$, Mauricio Duque ${ }^{6}$, Jorge Marin ${ }^{1}$, \\ Julian Aristizabal ${ }^{7}$, Jorge Velásquez ${ }^{8}$, Cesar Niño $^{8}$, and Hector Mazzetti ${ }^{9}$ \\ ${ }^{1}$ Clinica Las Americas \\ ${ }^{2}$ Montefiore Medical Center \\ ${ }^{3}$ Universidade de São Paulo Instituto do Coração \\ ${ }^{4}$ Centro Cardiovascular Universitario \\ ${ }^{5}$ Montefiore Hospital and Medical Center \\ ${ }^{6}$ Universidad CES \\ ${ }^{7}$ Clinica Las Vegas \\ ${ }^{8}$ Clinica Somer \\ ${ }^{9}$ Sanatorio de la Trinidad de San Isidro
}

May 10, 2021

\begin{abstract}
Background: Transvenous lead extraction (TLE) is standard of care for the management of patients with cardiac implantable electronic device infection or lead related complications. Currently, objective data on TLE in Latin America is lacking. Objective: To describe the current practice standards in Latin American centers performing TLE. Methods: An online survey was sent through the mailing list of the Latin American Heart Rhythm Association (LAHRS). Online reminders were sent through the mailing list; duplicate answers were discarded. The survey was available for one month, after which no more answers were accepted. Results: A total of 48 answers were received, from 44 different institutions (39.6\% from Colombia, $27,1 \%$ from Brazil), with most respondents (83\%) being electrophysiologists. Twenty-nine institutions (66\%) performed less than 10 lead extractions/year, with 7 (15\%) institutions not performing lead extraction. Although most institutions in which lead extraction is performed reported using several tools, mechanical rotating sheaths were cited as the main tool (73\%), 13.5\% reported the use of mechanical extraction sheaths and only $13.5 \%$ reporting the use of laser sheaths. Management of infected leads was performed according to current guidelines. Conclusion: This survey is the first attempt to provide information on TLE procedures in Latin America and could provide useful information for future prospective registries. According to our results, the number of centers performing high volume lead extraction in Latin America is smaller than that reported in other continents, with most interventions performed using mechanical tools. Future prospective registries assessing acute and long-term success are needed.
\end{abstract}

\section{Introduction}

Over the last decades, the number of cardiac implantable electronic devices (CIED) implanted has steadily increased as technological advances have allowed for a greater number of conditions to be treated with CIEDs $(1,2)$. Long-term CIED implants are usually associated with a need to add or replace existing electrodes due to lead failure or need for device upgrade, with many patients having abandoned leads which increase the risk of infection and venous thrombosis. Moreover, implants are being performed in older patients with more comorbidities $(3,4)$. This increase in implants and the use of devices in older, sicker patients have led to a higher number of device related infection and lead related complications (5,6). As such, there is an 
increasing need for lead extraction procedures $(7,8)$. Although technological advances have allowed for safer and easier percutaneous lead extraction procedures, access to different tools and the high costs limit in many cases their widespread availability and as such their use varies in different institutions and countries.

Although several centers in Latin America perform lead extraction, objective data on current practice is lacking. In order to obtain information on the current lead extraction practice in Latin America, we performed this survey.

\section{Methods}

In this descriptive cross-sectional observational study, physicians working at electrophysiology services in Latin American countries were sent a 16-question online survey (table 1) created using a commercial online platform (Google Forms@) were contacted using the Latin American Heart Rhythm Society (LAHRS) directory. Additional invitations were sent through national cardiology medical associations. Information regarding city and country of origin, hospital center, participation in lead extraction procedures, operator experience, number of procedures per year, available tools and procedural characteristics was collected. If a respondent answered that extractions were not performed in his/her institution, no further questions regarding lead extraction procedures were made. The online survey was available from October 1 to October $31^{\text {st }}$, 2020. All duplicate answers were eliminated. Results are presented as absolute frequencies and percentages for categorical variables.

\section{Results}

The survey was answered by 48 participants from 44 different institutions spanning 8 different countries. Transvenous lead extraction (TLE) was performed in $85 \%$ of these institutions. Most respondents were from Colombia (39.6\%) and Brazil (27.1\%) (figure 1A); $82 \%$ of respondents were electrophysiologists (figure 1B) with $71 \%$ of respondents having more than 10 years of experience (figure 1C).

Procedural characteristics

Forty-one respondents (85\%) performed lead extractions, with most institutions (66\%) performing less than 10 procedures per year and only $11 \%$ of of them preforming $>25$ procedures per year (figure $2 \mathrm{~A}$ ). Procedures were generally performed under general anesthesia (97\%). As expected, based on the respondent's specialty, electrophysiologists were reported to be in charge of lead extractions in $85 \%$ of cases (figure 2B). Out of the 38 institutions in which lead extraction was performed, laser powered sheaths were available in only 5 institutions (13\%), while mechanical tools (either mechanical dilators or rotating sheaths) were used by most respondents (figure 2C). Regarding safety measures and intra-procedural monitoring (figure 2D), the BRIDGE balloon (Phillips, San Diego, CA) was used in 5 institutions (13\%), with transesophageal echocardiography (TEE) and intracardiac echocardiography (ICE) used in $15(39 \%)$ and $6(16 \%)$ institutions, respectively. Most respondents reported a cardiovascular surgeon was either in the procedure room (46\%) or on call within the institution (46\%).

Proposed clinical scenarios

Answers to proposed clinical scenarios (including high-risk lead extraction procedures the management CIED pocked infections and malfunctioning leads) were received from the 42 respondents who performed lead extraction. Although the majority of respondents $(30,71 \%)$ had no specific clinical scenarios in which lead extraction would be avoided, $10(24 \%)$ respondents reported avoiding lead extraction in patients over 80 years of age (3A). In cases of device related pocket infection, total capsulectomy was performed by $24(57 \%)$, with 9 (24\%) reporting not performing capsulectomy (figure 3B). In pacing dependent patients with isolated pocket infection (figure 3C), a new device was implanted only after consultation with the infectious disease specialist by $18(43 \%)$ of respondents, with 5 respondents (12\%) reporting implantation of a new device during the same procedure. In patients with malfunctioning pacemaker or ICD leads, lead abandonment within the generator pocket (with implantation of a new lead) was routinely performed by 3 respondents $(7 \%)$, while $30(71 \%)$ respondents basing their decision on a case-by-case basis after analyzing individual patient characteristics and comorbidities (figure 3D) 


\section{Discussion}

During the last two decades, the number of CIEDs implanted has increased steadily as technological advances have allowed a wider range of conditions to be treated with implantable devices. Moreover, the number of dual chamber vs single chamber pacemakers, along with the use of cardiac resynchronization devices has also increased (9-12). As such, not only has the number of CIED implantations but also the number of implanted leads has increased. This has led to a higher number of lead related complications, mainly infection, and a greater number of TLE procedures performed.

This is the first Latin American survey on TLE. Although smaller than previous surveys (13-15), answers were received from 48 respondents from 44 different institutions ( 8 different countries). As such the survey provides valuable information on the current practice of lead extraction in our continent. Importantly, although TLE is performed in several institutions in Latin America, most (66\%) report a low procedural volume (i.e., less than 10 procedures per year). This percentage is higher than that reported in the US (in which less than $20 \%$ of institutions performed $<10$ lead extraction procedures per year) and the first European lead extraction survey (in which $40 \%$ of institutions performed $<10$ procedures each year)(15) demonstrating a low use of TLE in the continent. Several possible reasons exist for this finding: high cost of lead extraction tools, a perceived higher risk for complications (as evidenced by a reluctance to perform TLE in patients older than 80 years old in $22 \%$ of respondents), and a lack of proper training/knowledge (16). Unfortunately, the number of implant procedures and the infection rate in Latin America is currently unknown but taking into consideration European guidelines which estimate the need of TLE as 1.5 times the infection rate (17), a significantly higher number of lead extraction procedures is expected. Lack of public financing in many Latin American countries has a significant impact on CIED use and waiting times (18), and as such should also impact the use of TLE techniques. Implementation of training programs, along with a reduction in costs associated with TLE tools could have positive impact on the number of lead extraction cases performed. Taking into consideration that low procedural volume (defined in the ELECTRa registry as less than 30 lead extraction procedures per year) is associated with a significant increase in procedural mortality, efforts should be made to increase training and to perform lead extraction procedures only in high volume centers (19).

Although most institutions (73\%) used mechanical rotating sheaths (which is similar to what was found in the European survey)(13), $13.5 \%$ of institutions reported the use of laser sheaths. This is particularly surprising in latin america, taking into consideration the higher cost of laser sheaths (20), with procedural success rates similar to those reported with current mechanical rotating sheaths (21-23). In fact, in the recently published PROMET study laser sheaths were almost entirely abandoned in favor of mechanical rotating sheaths due to lower costs and similar effectiveness (24). Regarding safety measures, cardiac surgeons were included (either in room or within the institution) by $92 \%$ of respondents, similar to what has been described in the ILEEM survey (25) but higher than reported in the US survey (in which a surgeon was not identified in $25 \%$ of lead extraction procedures)(14). Importantly, other safety measures including TEE (39\%) ICE (16.2\%) and the BRIDGE balloon (13.5\%) were used in a significant number of institutions.

Regarding the proposed clinical scenarios, the number of respondents who perform complete capsulectomy in CIED related infection is lower than that reported in a recent worldwide survey $(57 \%$ vs $76 \%)(26)$. This is in line with the most recent guidelines, in which capsulectomy is not recommended as routine practice (27). Elderly patients (i.e., those older than 80 years of age) were considered to be at high risk of procedure related complications and $24 \%$ of respondents routinely avoided extraction in this patient population. Indeed, extraction procedures in elderly patients have been found to be significantly associated with a higher periprocedural mortality $(2.5 \%$ in $18-44$ years compared to $5.3 \%$ in $85+$ years, $\mathrm{P}<0.001)$ in the US (28). Finally, regarding malfunctioning leads, $71 \%$ of respondents would base their decision (i.e., lead abandonment or extraction) on individual patient characteristics. This is similar to what has been described in previous European surveys, in which malfunctioning lead management was strongly determined by patient's age, the presence of damaged leads and lead dwelling time (29).

Recently, the results of the largest prospective lead extraction registry in Latin America were published, 
demonstrating the safety and effectiveness of lead extraction in a large volume center (30). Future efforts should thus be focused on increasing the number of centers performing high volume lead extraction, as this study demonstrates results comparable to those published in literature can be achieved.

\section{Limitations}

Our results have limitations to be considered. Although the survey was sent using the LAHRS database, it is possible that several practitioners from high volume centers failed to answer the survey. Additionally, since the survey was sent in English, this could have also limited the number of possible physicians willing to answer the survey. This could have reduced the number of answers received. Finally, most answers were received from Colombia and Brazil (66.7\% of total answers), and as such other countries may be under-represented even though this type of procedures are known to be performed.

\section{Conclusions}

This survey is the first attempt to obtain information on current practices involving TLE in Latin America. According to our results, TLE is performed in select centers in Latin America, with most centers having a low procedural volume ( $<10$ procedures per year). As observed in other surveys, mechanical rotational sheaths are the most frequently tool used, with current management techniques closely resembling what is described in current guidelines. Results from this survey could be used as guidance towards the creation of a future prospective registry which could allow comparison of clinical outcomes using different extraction techniques and the implementation of quality improvement strategies.

Tables and Figures

Name of the institution you most of the time work in (OQ) In which country do you live in? (MC, SA) What is your specie

Table 1: Survey questions and type of answer expected. Abbreviations: OQ: open question; MC: Multiple choice; SA: single
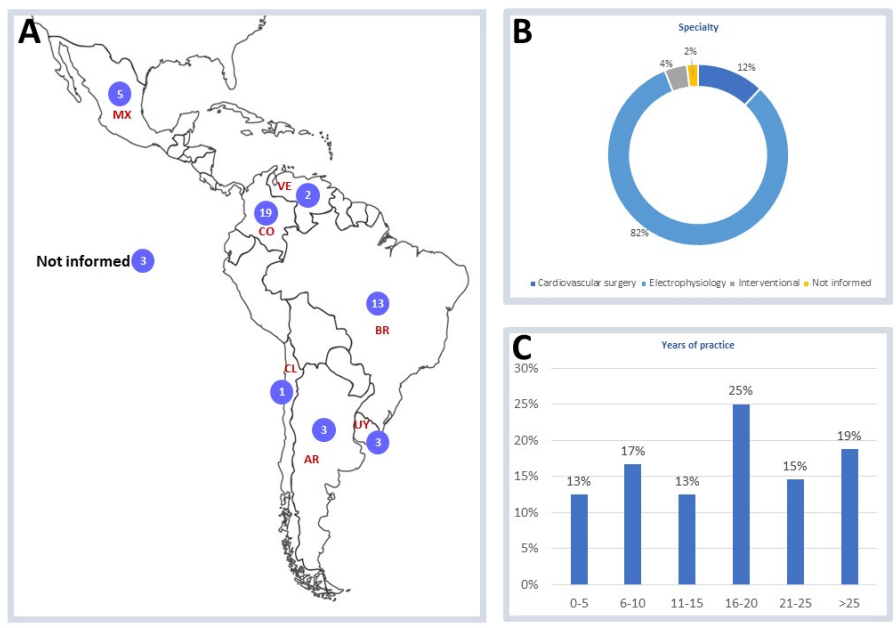

Figure 1. A: Number of respondents per country.Although answers from several countries were received, the majority of respondents were either from Colombia or Brazil. Most respondents were electrophysiologists (B), with $70 \%$ having more than 10 years of experience. Abbreviations: MX: Mexico; CO: Colombia; VE: Venezuela; BR: Brazil; CL: Chile; AR; Argentina; UY: Uruguay. 


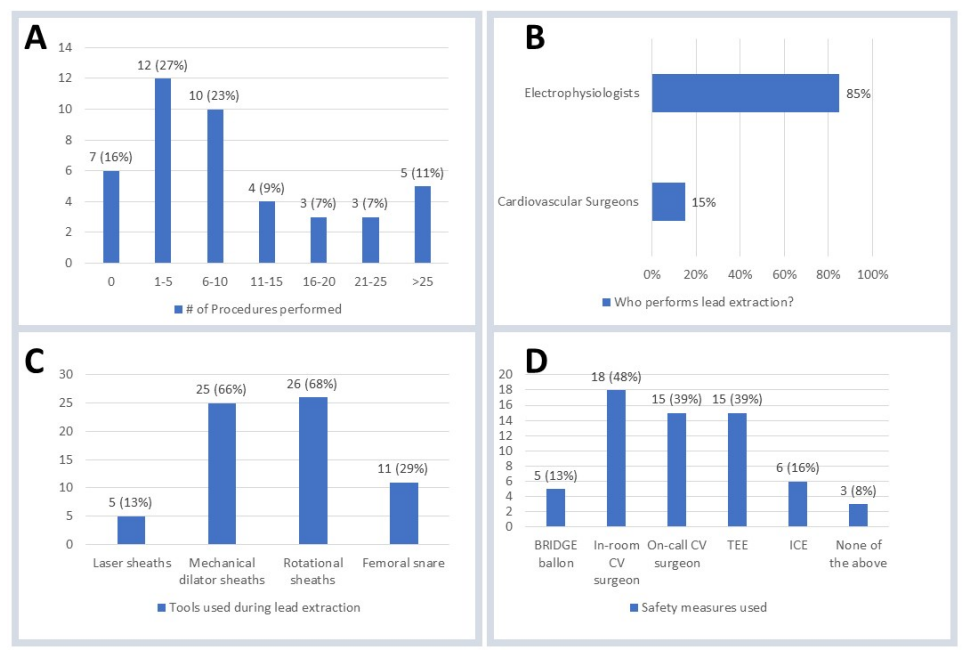

Figure 2. Information from 44 different institutions was obtained. A: Number of procedures performed per institution. B: In most institutions, lead extraction is performed by electrophysiologists. C: Most institutions rely on mechanical lead extraction tools, with only 5 institutions (13\%) using laser powered sheaths. D: Safety measures used during lead extraction procedures. Eighty-seven percent of respondents reported cardiovascular surgeons were present either in room or on call within the institution. Abbreviations: CV: cardiovascular; TEE: transesophageal echocardiography; ICE: intracardiac echocardiography.
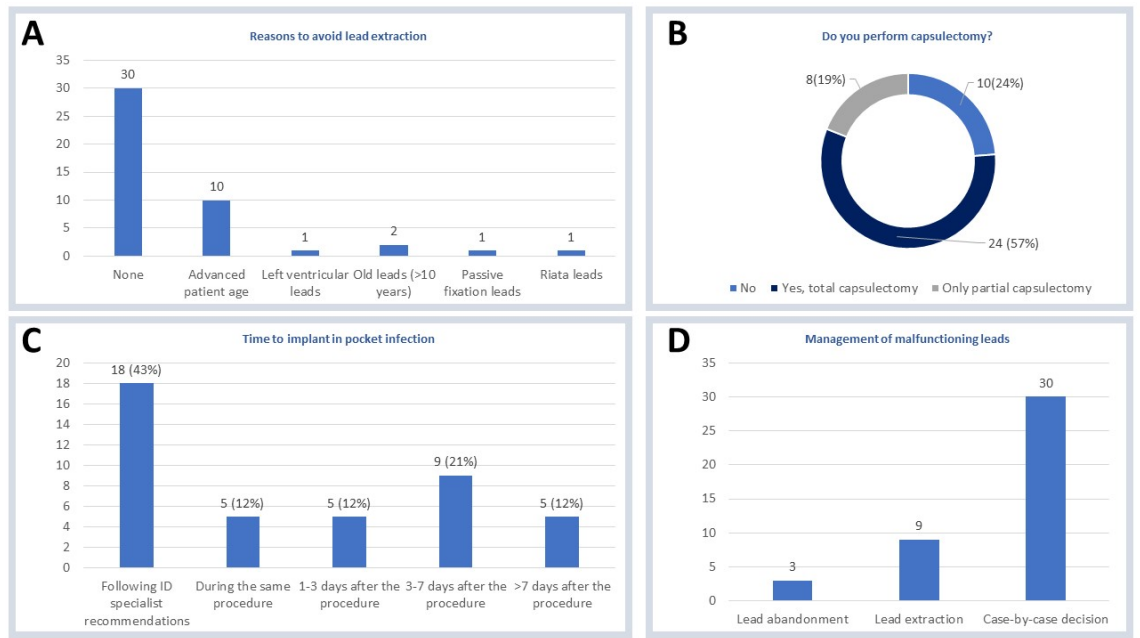

Figure 3. Respondents' answers to proposed clinical scenarios, including reasons to avoid lead extraction (A), use of capsulectomy during lead extraction procedures in device related infections (B), time to reimplantation in pacemaker dependent patients who have isolated CIED pocket infection (i.e., without bacteremia or endocarditis) (C) and approach to patients with malfunctioning pacemaker or ICD leads. Abbreviations: ID: infectious disease; CIED: cardiac implantable electronic device; ICD: implantable cardioverter defibrillator.

\section{References}

1. Foo FS, Stiles MK, Lee M et al. Ten year trends in cardiac implantable electronic devices in New Zealand: a national data linkage study (ANZACS-QI 51). Internal medicine journal 2020. 
2. Levine PA, Stanton MS, Sims JJ. High quality performance of pacemakers and implantable defibrillators. Pacing and clinical electrophysiology : PACE 2002;25:1667-9.

3. Lee JH, Lee SR, Choi EK et al. Temporal Trends of Cardiac Implantable Electronic Device Implantations: a Nationwide Population-based Study. Korean circulation journal 2019;49:841-852.

4. Yokoshiki H, Shimizu A, Mitsuhashi T et al. Trends in the use of implantable cardioverter-defibrillator and cardiac resynchronization therapy device in advancing age: Analysis of the Japan cardiac device treatment registry database. Journal of arrhythmia 2020;36:737-745.

5. Catanchin A, Murdock CJ, Athan E. Pacemaker infections: a 10-year experience. Heart, lung \& circulation 2007;16:434-9.

6. Voigt A, Shalaby A, Saba S. Continued rise in rates of cardiovascular implantable electronic device infections in the United States: temporal trends and causative insights. Pacing and clinical electrophysiology : PACE 2010;33:414-9.

7. Bongiorni MG, Burri H, Deharo JC et al. 2018 EHRA expert consensus statement on lead extraction: recommendations on definitions, endpoints, research trial design, and data collection requirements for clinical scientific studies and registries: endorsed by APHRS/HRS/LAHRS. Europace : European pacing, arrhythmias, and cardiac electrophysiology : journal of the working groups on cardiac pacing, arrhythmias, and cardiac cellular electrophysiology of the European Society of Cardiology 2018;20:1217.

8. Kusumoto FM, Schoenfeld MH, Wilkoff BL et al. 2017 HRS expert consensus statement on cardiovascular implantable electronic device lead management and extraction. Heart rhythm : the official journal of the Heart Rhythm Society 2017;14:e503-e551.

9. Larsen PD, Kerr AJ, Hood M et al. Pacemaker Use in New Zealand - Data From the New Zealand Implanted Cardiac Device Registry (ANZACS-QI 15). Heart, lung \& circulation 2017;26:235-239.

10. Greenspon AJ, Patel JD, Lau E et al. 16-year trends in the infection burden for pacemakers and implantable cardioverter-defibrillators in the United States 1993 to 2008. Journal of the American College of Cardiology 2011;58:1001-6.

11. Greenspon AJ, Patel JD, Lau E et al. Trends in permanent pacemaker implantation in the United States from 1993 to 2009: increasing complexity of patients and procedures. Journal of the American College of Cardiology 2012;60:1540-5.

12. Zecchin M, Torre M, Carrani E et al. Seventeen-year trend (2001-2017) in pacemaker and implantable cardioverter-defibrillator utilization based on hospital discharge database data: An analysis by age groups. European journal of internal medicine 2020.

13. Bongiorni MG, Blomström-Lundqvist C, Kennergren C et al. Current practice in transvenous lead extraction: a European Heart Rhythm Association EP Network Survey. Europace : European pacing, arrhythmias, and cardiac electrophysiology : journal of the working groups on cardiac pacing, arrhythmias, and cardiac cellular electrophysiology of the European Society of Cardiology 2012;14:783-6.

14. Henrikson CA, Zhang K, Brinker JA. A survey of the practice of lead extraction in the United States. Pacing and clinical electrophysiology : PACE 2010;33:721-6.

15. van Erven L, Morgan JM. Attitude towards redundant leads and the practice of lead extractions: a European survey. Europace : European pacing, arrhythmias, and cardiac electrophysiology : journal of the working groups on cardiac pacing, arrhythmias, and cardiac cellular electrophysiology of the European Society of Cardiology 2010;12:275-6.

16. Rao A, Garner D, Starck C et al. Knowledge gaps, lack of confidence, and system barriers to guideline implementation among European physicians managing patients with CIED lead or infection complications: a European Heart Rhythm Association/European Society of Cardiology educational needs assessment survey. 
Europace : European pacing, arrhythmias, and cardiac electrophysiology : journal of the working groups on cardiac pacing, arrhythmias, and cardiac cellular electrophysiology of the European Society of Cardiology 2020;22:1743-1753.

17. Deharo JC, Bongiorni MG, Rozkovec A et al. Pathways for training and accreditation for transvenous lead extraction: a European Heart Rhythm Association position paper. Europace : European pacing, arrhythmias, and cardiac electrophysiology : journal of the working groups on cardiac pacing, arrhythmias, and cardiac cellular electrophysiology of the European Society of Cardiology 2012;14:124-34.

18. Lau CP, Tse HF, Mond HG. The impact of reimbursement on the usage of pacemakers, implantable cardioverter defibrillators and radiofrequency ablation. Journal of interventional cardiac electrophysiology : an international journal of arrhythmias and pacing 2006;17:177-81.

19. Sidhu BS, Gould J, Bunce C et al. The effect of centre volume and procedure location on major complications and mortality from transvenous lead extraction: an ESC EHRA EORP European Lead Extraction ConTRolled ELECTRa registry subanalysis. Europace : European pacing, arrhythmias, and cardiac electrophysiology : journal of the working groups on cardiac pacing, arrhythmias, and cardiac cellular electrophysiology of the European Society of Cardiology 2020;22:1718-1728.

20. Starck CT, Rodriguez H, Hürlimann D et al. Transvenous lead extractions: comparison of laser vs. mechanical approach. Europace : European pacing, arrhythmias, and cardiac electrophysiology : journal of the working groups on cardiac pacing, arrhythmias, and cardiac cellular electrophysiology of the European Society of Cardiology 2013;15:1636-41.

21. Cay S, Ozeke O, Ozcan F, Topaloglu S, Aras D. Comparison of two types of rotational mechanical dilatator sheath: Evolution(@) and TightRail(). Pacing and clinical electrophysiology : PACE 2019;42:1226-1235.

22. Migliore F, Testolina M, Sagone A et al. Multicenter experience with the Evolution RL mechanical sheath for lead extraction using a stepwise approach: Safety, effectiveness, and outcome. Pacing and clinical electrophysiology : PACE 2019;42:989-997.

23. Qin D, Chokshi M, Sabeh MK et al. Comparison between TightRail rotating dilator sheath and GlideLight laser sheath for transvenous lead extraction. Pacing and clinical electrophysiology : PACE 2021.

24. Starck CT, Gonzalez E, Al-Razzo O et al. Results of the Patient-Related Outcomes of Mechanical lead Extraction Techniques (PROMET) study: a multicentre retrospective study on advanced mechanical lead extraction techniques. Europace : European pacing, arrhythmias, and cardiac electrophysiology : journal of the working groups on cardiac pacing, arrhythmias, and cardiac cellular electrophysiology of the European Society of Cardiology 2020;22:1103-1110.

25. Starck CT, Bracke F, Delnoy PP et al. ILEEM-survey on the Heart Team approach and team training for lead extraction procedures. Cardiology journal 2020.

26. Traykov V, Bongiorni MG, Boriani G et al. Clinical practice and implementation of guidelines for the prevention, diagnosis and management of cardiac implantable electronic device infections: results of a worldwide survey under the auspices of the European Heart Rhythm Association. Europace : European pacing, arrhythmias, and cardiac electrophysiology : journal of the working groups on cardiac pacing, arrhythmias, and cardiac cellular electrophysiology of the European Society of Cardiology 2019.

27. Blomstrom-Lundqvist C, Traykov V, Erba PA et al. European Heart Rhythm Association (EHRA) international consensus document on how to prevent, diagnose, and treat cardiac implantable electronic device infections-endorsed by the Heart Rhythm Society (HRS), the Asia Pacific Heart Rhythm Society (APHRS), the Latin American Heart Rhythm Society (LAHRS), International Society for Cardiovascular Infectious Diseases (ISCVID) and the European Society of Clinical Microbiology and Infectious Diseases (ESCMID) in collaboration with the European Association for Cardio-Thoracic Surgery (EACTS). European journal of cardio-thoracic surgery : official journal of the European Association for Cardio-thoracic Surgery 2020;57:e1-e31. 
28. Sridhar AR, Lavu M, Yarlagadda V et al. Cardiac Implantable Electronic Device-Related Infection and Extraction Trends in the U.S. Pacing and clinical electrophysiology : PACE 2017;40:286-293.

29. Grazia Bongiorni M, Dagres N, Estner H, Pison L, Todd D, Blomstrom-Lundqvist C. Management of malfunctioning and recalled pacemaker and defibrillator leads: results of the European Heart Rhythm Association survey. Europace : European pacing, arrhythmias, and cardiac electrophysiology : journal of the working groups on cardiac pacing, arrhythmias, and cardiac cellular electrophysiology of the European Society of Cardiology 2014;16:1674-8.

30. Costa R, Silva KRD, Crevelari ES et al. Effectiveness and Safety of Transvenous Removal of Cardiac Pacing and Implantable Cardioverter-defibrillator Leads in the Real Clinical Scenario. Arquivos brasileiros de cardiologia 2020;115:1114-1124. 